\title{
Nota sobre os diferenciais de salários no Brasil: uma investigação empírica sob a perspectiva da Teoria da Segmentação
}

\author{
Eliane Araújo* \\ Adriana Evarini** \\ Maria de Fátima Garcia ${ }^{* * *}$ \\ Elisangela Luzia Araújo ***
}

\begin{abstract}
RESUMO - O presente artigo empreende uma análise sobre os determinantes dos diferenciais de salários no Brasil, considerando as várias categorias ocupacionais, típicas dos segmentos primário e secundário do mercado de trabalho. São utilizados modelos de regressão quantílica, aplicados aos dados da PNAD de 2009, para aferir a importância de variáveis tais como educação, idade, cor e gênero na determinação destes diferenciais. Em consonância com a hipótese de heterogeneidade do mercado de trabalho, apontada pela Teoria da Segmentação, os resultados do artigo sugerem que: i) a educação é menos importante para determinar o rendimento naqueles segmentos de trabalho mais precários, quais sejam as categorias dos Trabalhadores dos Serviços e da Produção; ii) os coeficientes das variáveis cor e gênero apontam a existência de preconceito no mercado de trabalho, que é maior nas categorias ocupacionais com maior potencial de rendimento, isto é, os Profissionais das Ciências e das Artes e os Dirigentes; e, por fim, iii) a variável idade é a menos significativa para determinar o rendimento nas diferentes ocupações.
\end{abstract}

Palavras-chave: Teoria da Segmentação. Mercado de trabalho. Categorias ocupacionais.

\section{INTRODUÇÃO}

Os diferenciais de rendimentos do trabalho constituem um tema recorrente na literatura e sua discussão tem como arcabouço teórico basilar duas abordagens concorrentes, as quais buscam explicação das causas destes diferenciais: a Teoria do Capital Humano e a Teoria da Segmentação. A primeira, em sua versão mais acabada, é atribuída a Schultz $(1960,1961)^{1}$ e * Doutora em Economia pela Universidade Federal do Rio de Janeiro. É professora adjunta da Universidade Estadual de Maringá e membro do Grupo de Pesquisa Tecnologia, Trabalho e Desenvolvimento. Endereço eletrônico: elianedearaujo@yahoo.com.br.

** Mestre em Economia pela Universidade Estadual de Londrina. É membro do Grupo de Pesquisa Tecnologia, Trabalho e Desenvolvimento. Endereço eletrônico: adrianaevarini@hotmail.com.

*** Doutora em Economia pela Universidade Federal de Pernambuco. É professora associada da Universidade Estadual de Maringá e membro do Grupo de Pesquisa Tecnologia, Trabalho e Desenvolvimento. Endereço eletrônico: mfgarcia@uem.br.

**** Mestre em Economia pela Universidade Estadual de Maringá. É doutoranda da Universidade Federal do Rio Grande do Sul e membro do Grupo de Pesquisa Tecnologia, Trabalho e Desenvolvimento. Endereço eletrônico: elisangelal_araujo@yahoo.com.br.

1 Schultz (1960) define capital humano como o conjunto de habilidades cognitivas, adquiridas por meio do investimento em educação formal (escolaridade e educação do mais alto nível), treinamento realizado no próprio local de trabalho (on the job training), migração, saúde e informação. Deste modo, atributos técnicos podem ser alterados 
Becker (1964), e toma por pressuposto que o mercado de trabalho é contínuo, sugerindo uma perfeita mobilidade dos trabalhadores das classes de salários mais baixos para as de salários mais altos, possibilitada pela incorporação crescente de capital humano.

As análises apoiadas na Teoria do Capital Humano privilegiam o lado da oferta de trabalho, ao enfatizar as características individuais dos trabalhadores na determinação tanto das oportunidades, quanto da renda auferida no trabalho. Deste modo, maiores salários são compatíveis com maior nível de investimento em capital humano e a hierarquia salarial é resultado de tais diferenças (MULS, 1999). As variações no capital humano dos indivíduos vão determinar sua ocupação e rendimento, já que este é considerado um ativo, cuja rentabilidade é uma função de quanto investimento foi realizado para a sua aquisição.

Em Lima (1980), são elaboradas diversas críticas à Teoria do Capital Humano. Uma delas refere-se ao fato de que, ao supor a existência de uma relação entre decisões deliberadas de investimento em capital humano, produtividade e rendimento, esta teoria impede que se apontem, de forma apropriada, as razões que causam as diferenças nas rendas individuais. Ocorre que diferentes pessoas possuem diferentes tipos e quantidades de capital físico e humano, que não resultam apenas da educação formal.

Outra crítica apontada pelo autor está relacionada à utilização da taxa de retorno de investimento em autovalor do indivíduo. Um dos problemas é o caráter estático desse modelo, haja visto que as taxas de retorno do investimento em educação estão sempre mudando. $O$ outro questionamento é proveniente das evidências empíricas que mostram que a importância da taxa de retorno para as decisões de investimento em educação é praticamente desprezível. Por fim, consideram-se os argumentos que sugerem que as escolas não geram meramente um produto cognitivo, mas diferentes tipos de socialização, sinais e credenciais que influenciam os rendimentos futuros dos estudantes.

Foi o conjunto dessas críticas que levou ao desenvolvimento de uma segunda abordagem, anteriormente referida: a Teoria da Segmentação ou da Dualidade do Mercado de Trabalho que, ao invés de enfatizar a educação na determinação da renda, preocupa-se com o local em que a renda é gerada: o posto de trabalho. Nesta segunda abordagem, o mercado de trabalho é descontínuo, apresentando dois segmentos, primário e secundário, que se distinguem segundo as condições de trabalho e o tipo de ocupação inerente a cada um destes.

No segmento primário abrigam-se os empregos regidos por relações de trabalho formais e estáveis próprias das grandes empresas oligopolistas, e ainda pode ser compartimentado em dois: primário independente ou criativo, onde se encontram os trabalhadores do topo por meio do investimento no homem, o qual seria suscetível à mudança técnica. 
da pirâmide salarial, os quais exercem funções inerentes às tomadas de decisões das grandes empresas e requerem capacidade de iniciativa própria e criatividade. O outro subsegmento é o primário dependente ou rotineiro, onde se encontram os trabalhadores que exercem ocupações rotineiras do chão de fábrica, dos escritórios e burocráticas da administração pública direta e indireta (CACCIAMALI, 1978).

No segmento primário, as ocupações e as condições de trabalho são tais que os níveis salariais são mais elevados vis-à-vis os níveis salariais vigentes no segmento secundário, onde as ocupações requerem um mínimo de qualificação e as condições de inserção da mão de obra são as mais precárias (LIMA, 1980; CACCIAMALI, 1978).

Tais segmentos constituem o locus de inserção das ocupações segundo fatores históricos que permitem a separação dos trabalhadores entre os empregos de alta e baixa produtividade (bons e maus empregos, de mercado primário ou secundário), ou aqueles restritos às pessoas das classes dominantes e das classes periféricas (LIMA, 1980). Nessa perspectiva, os salários são influenciados pelas condições inerentes a cada um dos segmentos, primário e secundário, em que as ocupações estão inseridas. Tais condições exprimem diferentes correlações de forças entre empregadores e empregados e, por conseguinte, diferenciais de salários. Ainda de acordo com o referido autor, as empresas são moldadas por valores sociais e culturais, que podem facilitar ou criar barreiras às oportunidades de trabalho, o que favorece o surgimento das disparidades salariais. Assim, desfaz-se a relação de causação direta entre educação (e treinamento) e salários, conforme postulado pela Teoria do Capital Humano, não obstante a educação exerça papel preponderante na inserção dos trabalhadores, segundo suas competências, nos diferentes segmentos do mercado de trabalho (LIMA, 1980).

Ainda nessa perspectiva, é plausível admitir que, dentro de cada segmento, a relação referida possa confirmar-se, pois se admite a mobilidade de trabalhadores intrassegmento, mas não entre estes e tampouco entre o primário dependente e o independente. Acresce que cada segmento introjeta condições econômicas, sociais, organizacionais e históricas peculiares, que definem a natureza e o tipo de ocupação aí inserida. Em outras palavras, se a Teoria do Capital Humano sugere que níveis mais altos de educação correspondem a níveis mais altos de renda, a Teoria da Segmentação admite que se as ocupações forem classificadas segundo sua natureza, o papel da educação na determinação do rendimento tende a diminuir, sobretudo naqueles segmentos caracterizados por maior rotatividade da mão de obra, salários relativamente baixos, piores condições de trabalho, baixa produtividade do trabalho e estagnação tecnológica.

É no âmbito da Teoria da Segmentação que se insere o presente estudo, tendo em vista dois objetivos: i) averiguar, empiricamente, para seis categorias ocupacionais, inerentes aos 
segmentos primário e secundário do mercado de trabalho, a presença de diferenciais de salários; ii) analisar se (e até que ponto) variáveis tais como cor, gênero, idade e educação são determinantes dos diferenciais de salários dentro de cada uma destas categorias ocupacionais. Para se alcançarem tais objetivos, o presente estudo utiliza-se da metodologia de regressões quantílicas, aplicada aos dados da PNAD para o ano de 2009.

$\mathrm{Na}$ sequência desta introdução, são apresentadas algumas evidências empíricas sobre os determinantes dos diferenciais de salários no Brasil, os procedimentos metodológicos e a discussão dos resultados das regressões quantílicas aplicadas aos diferentes grupos ocupacionais identificados.

\section{ANÁLISE EMPÍRICA DO DIFERENCIAL DE RENDIMENTOS NAS OCUPA- ÇÕES EM 2009}

\subsection{BASE DE DADOS E METODOLOGIA}

O presente estudo tem por base as informações coletadas na Pesquisa Nacional por Amostra de Domicílios (PNAD) de 2009. Esta pesquisa tem periodicidade anual desde 1971, sendo interrompida por ocasião dos Censos Demográficos de 1970, 1980, 1991 e 2000. Tratase de um levantamento anual, realizado por meio de uma amostra dos domicílios, que abrange todo o país. O procedimento metodológico adotado pelo IBGE implica que cada pessoa da amostra representa um determinado número de pessoas da população. Os dados individuais desse trabalho são fornecidos com o peso ou fator de expansão de cada indivíduo, que permite que os dados sejam elaborados ponderando-se cada observação pelo respectivo peso.

De acordo com as notas metodológicas do IBGE (2007), as ocupações foram especificadas segundo a Classificação Brasileira de Ocupações - Domiciliar (CBO). Consideram-se rendimentos de trabalho aqueles decorrentes dos pagamentos brutos mensais aos empregados, empregadores e autônomos advindos do trabalho principal. O trabalho principal é o trabalho único que a pessoa de dez ou mais anos de idade teve no período da pesquisa (semana de referência da PNAD), impondo-se outros critérios para casos especiais.

As ocupações estão divididas em seis grupos: Dirigentes (DIR), Profissionais das Ciências e das Artes (PCA), Técnicos de Nível Médio (TMED), Trabalhadores de Serviços Administrativos (SADM), Trabalhadores dos Serviços (SERV), Trabalhadores da Produção de bens e serviços industriais e de reparação e manutenção (TPROD).

Da perspectiva da Teoria da Segmentação do Mercado de Trabalho, é plausível admitir que as categorias DIR e PCA sejam próprias do segmento primário independente, enquanto as 
categorias TMED e SADM sejam próprias do primário dependente. As outras duas, SERV e TPROD, podem ser admitidas nos segmento primário dependente e no secundário.

Quanto à cor, as variáveis estudadas classificam-se em brancos e não brancos (amarelo, negro e pardo). Os grupos de escolaridade são definidos em quatro intervalos de períodos de estudo: de zero até 4 anos, de 5 até 8 anos, de 9 até 11 anos e de 12 ou mais anos de estudo. O perfil etário divide-se em três faixas etárias: de 16 até 24 anos, de 25 até 39 anos e de 40 até 65 anos.

Valendo-se da metodologia de regressões quantílicas proposta por Machado e Mata (2005), são estimados dois modelos. O primeiro tem por objetivo identificar se a ocupação é importante para explicar o rendimento nos diferentes quantis de renda, conforme a equação seguinte:

$$
\ln w=\alpha+\beta_{1} \text { escolaridade }+\beta_{2} \text { idade }+\beta_{2} \text { idade }{ }^{2}+\beta_{4} \text { branco }+\beta_{5} \text { fem }+\beta_{6} \text { ocupação }+\varepsilon
$$

Na Equação (1), a variável dependente é o logaritmo natural do rendimento do trabalho principal (lnw) e as variáveis explicativas são: nível de escolaridade (escolaridade), idade (idade) e idade ao quadrado (idade ${ }^{2}$ ), sexo (fem), cor (branco) e ocupação (ocupação).

As variáveis binárias nesse modelo são: fem, branco e ocupação. Ou seja, fem assume valor igual a 1 quando mulher e 0 caso contrário. A variável branco assume valor igual a 1 quando a cor é branca e 0 quando é não branco (negro, amarelo e pardo) e, de forma análoga, foram criadas dummies para as ocupações estudadas, sendo que ocupação assume valor igual a 1 quando Dirigentes e 0 nas demais ocupações. O modelo foi estimado para indivíduos de 0 a 17 anos de estudo e com perfil etário de 10 a 60 anos de idade.

O segundo modelo estima uma equação de rendimentos para cada uma das ocupações, visando identificar como cada variável determinante do rendimento se manifesta nas diferentes ocupações, ao longo dos quantis de renda. A Equação (2) ilustra esse modelo, em que $i$ representa as diferentes categorias ocupacionais:

$$
\operatorname{lnw}_{i}=\alpha+\beta_{1} \text { escolaridade }_{i}+\beta_{2} \text { idade }_{i}+\beta_{2} \text { idade }_{i}^{2}+\beta_{4} \text { branco }_{i}+\beta_{5} \text { fem }_{i}+\varepsilon_{i}
$$

\subsection{RESULTADOS E DISCUSSÃO}

Os resultados alcançados revelam que, independentemente do percentil analisado, o $\log$ do rendimento da ocupação Dirigentes e Profissionais das Ciências e das Artes é maior que o das outras ocupações, sugerindo a existência de desigualdade nos rendimentos dos grupos 
ocupacionais em todos os quantis de renda (Tabela 1).

TABELA 1 - RENDIMENTO NOS PERCENTIS POR OCUPAÇÃO

\begin{tabular}{l|r|r|rr|r|r}
\hline \multicolumn{1}{c}{ Ocupação } & p10 (lnw) & p25 (lnw) & med (lnw) & p75 (lnw) & p90 (lnw) \\
\hline DIR & 6,397 & 6.908 & 7,484 & 8,006 & 8,517 \\
PCA & 5,991 & 6.551 & 7,244 & 7,937 & 8,517 \\
TMED & 5,940 & 6,215 & 6,685 & 7,313 & 7,863 \\
SADM & 5,940 & 5,991 & 6,370 & 6,795 & 7,313 \\
SERV & 4.942 & 5,704 & 5,940 & 6,261 & 6,685 \\
TPROD & 5.521 & 5,940 & 6,310 & 6,685 & 7,090 \\
\hline
\end{tabular}

FONTE: Elaboração própria a partir dos dados da PNAD de 2009.

Para investigar a disparidade de renda em cada ocupação utiliza-se o cálculo do coeficiente de $\mathrm{Gini}^{2}$ e do índice de Theil ${ }^{3}$, cujos coeficientes podem variar de 0 a 1 , sendo que 0 exprime uma situação em que todos possuem uma mesma renda e 1 a situação na qual a renda se concentra em uma só pessoa, portanto, quanto mais próximo de 0 melhor a distribuição em cada ocupação, segundo os referidos indicadores. Os cálculos estão dispostos na Tabela 2.

TABELA 2 - ÍNDICE DE GINI E THEIL DO RENDIMENTO DAS OCUPAÇÕES

\begin{tabular}{lcr}
\hline \multicolumn{1}{c|}{ Ocupações } & Índice de Gini & Índice deTheil \\
\hline DIR & 0.494 & 0.474 \\
PCA & 0.516 & 0.485 \\
TMED & 0.469 & 0.409 \\
SADM & 0.379 & 0.285 \\
SERV & 0.371 & 0.292 \\
TPROD & 0.297 & 0.297 \\
TOTAL & 0.382 & 0.297 \\
\hline
\end{tabular}

FONTE: Elaboração própria a partir dos dados da PNAD de 2009.

Tanto o índice de Gini como o índice de Theil mostram que a categoria de ocupação dos Profissionais das Ciências e das Artes é aquela caracterizada por maior disparidade de rendimentos, seguida pela ocupação intitulada Dirigentes.

Vale destacar que os Profissionais das Ciências e das Artes têm a segunda maior renda. Entre as ocupações menos desiguais, no que se refere ao rendimento do trabalho principal, estão os Trabalhadores dos Serviços e os Trabalhadores da Produção. Observa-se, portanto, um contraste entre o potencial de rendimentos e a igualdade de renda entre as ocupações, isto é, aquelas ocupações que são caracterizadas por maior rendimento médio também são marcadas por disparidades de renda mais elevadas entre os trabalhadores que as compõem.

Dando continuidade à análise empírica, a Tabela 3 traz a estimativa de uma equação

2 O índice de Gini mede a desigualdade de renda no país. Quanto mais próximo de 1 maior é a concentração de renda. Se igual a 1 , significa que um indivíduo concentra toda a renda da sociedade. Se igual a zero, significa que não existe concentração de renda (HOFFMANN, 1998).

3 O índice de entropia, T de Theil, também é uma medida da desigualdade de uma distribuição, que é nulo quando não houver desigualdade e tende ao infinito quanto maior a desigualdade. $\mathrm{O} T$ de Theil é mais sensível à mudanças na renda dos ricos ou no que ocorre na cauda superior da distribuição (HOFFMANN, 1998). 
de salários tradicional, que contempla as variáveis relevantes para explicar o diferencial de rendimento do trabalho. O objetivo desta regressão é investigar a importância da ocupação para a determinação do rendimento do trabalho, nos diferentes quantis de renda.

TABELA 3 - RESULTADO DAS REGRESSÕES QUANTÍLICAS

\begin{tabular}{lr|r|r|r|r}
\hline & \multicolumn{1}{c}{ Q10 } & \multicolumn{1}{c|}{ Q25 } & \multicolumn{1}{c|}{ Q50 } & \multicolumn{1}{c}{ Q75 } & \multicolumn{1}{c}{ Q90 } \\
\hline \multirow{2}{*}{ escolaridade } & 0.0990948 & 0.8449266 & 0.0889806 & 0.1038813 & 0.1184746 \\
& $(0.0007937)$ & $(0.0007844)$ & $(0.0004245)$ & $(0.0009736)$ & $(0.049023)$ \\
& {$[124.85]$} & {$[108.27]$} & {$[209.63]$} & {$[106.70]$} & {$[16.72]$} \\
idade & 0.0768145 & 0.0839145 & 0.0008507 & 0.0768145 & 0.0832987 \\
& $(0.0013123)$ & $(0.0010468)$ & $(0.0008944)$ & $(0.0008944)$ & $(0.0015166)$ \\
& {$[84.10]$} & {$[80.16]$} & {$[85.52]$} & {$[85.88]$} & {$[54.93]$} \\
idade^2 & -0.011977 & -0.0008672 & -0.000691 & -0.0006849 & -0.0007118 \\
& $(0.0000165)$ & $(0.0000136)$ & $(0.0000114)$ & $(0.0000111)$ & $(0.0000191)$ \\
branco & {$[-72.50]$} & {$[-63.72]$} & {$[-60.69]$} & {$[-61.79]$} & {$[-37.22]$} \\
& 0.218323 & 0.1985511 & 0.194946 & 0.2166925 & 0.22692 \\
fem & $(0.0092749)$ & $(0.0041447)$ & $(0.0051825)$ & $(0.0039321)$ & $(0.0064216)$ \\
& {$[23.54]$} & {$[65.94]$} & {$[37.62]$} & {$[55.11]$} & {$[35.34]$} \\
& -0.5466107 & -0.4377442 & -0.4099152 & -0.441436 & -0.4805526 \\
ocupação 1 & $(0.0073109)$ & $(0.0053026)$ & $(0.0046793)$ & $(0.0077162)$ & $(0.0072056)$ \\
& {$[-74.77]$} & {$[-547.90]$} & {$[-87.60]$} & {$[-57.21]$} & {$[-66.69]$} \\
& 0.4992435 & 0.5688844 & 0.6534937 & 0.6947339 & 0.736999 \\
& $(0.009869)$ & $(0.0086269)$ & $(0.0111286)$ & $(0.0136543)$ & $(0.0229132)$ \\
& {$[50.59]$} & {$[65.94]$} & {$[58.72]$} & {$[50.88]$} & {$[32.16]$} \\
\hline
\end{tabular}

FONTE: Elaboração própria a partir de dados da PNAD de 2009.

NOTA: Entre parênteses apresentam-se os desvios-padrão e entre colchetes a estatística T.

A Tabela 3 indica que a ocupação é fundamental na determinação dos diferenciais de rendimento e que esta variável torna-se mais importante nos quantis de renda mais elevados.

$\mathrm{Na}$ Tabela 4 encontram-se os resultados da estimativa da equação dos diferenciais de rendimentos para cada ocupação, dado o objetivo de apreender uma medida da importância das variáveis escolaridade, idade, sexo e cor em cada uma das ocupações delimitadas.

Observa-se, segundo a Tabela 4, que a educação é mais importante para explicar o rendimento nas ocupações dos Profissionais das Ciências e das Artes e dos Dirigentes nos quantis 10 e 25, e a partir do quantil 50 a educação torna-se mais importante na categoria dos Técnicos de Nível Médio do que na categoria Dirigentes. Nas ocupações dos Trabalhadores de Serviços Administrativos, Trabalhadores dos Serviços e Trabalhadores da Produção o coeficiente associado à educação é bem menor, sobretudo aquele referente ao grupo dos Trabalhadores dos Serviços.

Esse fato corrobora a tese apresentada pela Teoria da Segmentação do Mercado de Trabalho, que postula que se as ocupações forem classificadas segundo sua natureza, o papel da educação na determinação do rendimento tende a diminuir, especialmente naqueles segmentos caracterizados por maior rotatividade da mão de obra, salários relativamente baixos, piores condições de trabalho, baixa produtividade do trabalho e estagnação tecnológica. 
TABELA 4 - RESULTADO DAS REGRESSÕES QUANTÍLICAS POR OCUPAÇÃO

\begin{tabular}{lrrrrr|r|r}
\hline \multicolumn{1}{c}{ Lnw } & \multicolumn{1}{c}{ DIR } & \multicolumn{1}{c}{ PCA } & \multicolumn{1}{c}{ TME } & \multicolumn{1}{c}{ SAD } & \multicolumn{1}{c}{ SERV } & \multicolumn{1}{c}{ TPROD } \\
\hline \multirow{3}{*}{ escolaridade } & 0.0819893 & 0.189796 & 0.0815019 & 0.0518266 & 0.0669827 & 0.0693409 \\
& $(0.049023)$ & $(0.0066821)$ & $(0.003241)$ & $(0.0028463)$ & $(0.029044)$ & $(0.0024968)$ \\
& {$[16.72]$} & {$[28.40]$} & {$[25.15]$} & {$[18.21]$} & {$[26.51]$} & {$[27.77]$} \\
idade & 0.0801746 & 0.1050374 & 0.1035913 & 0.07639 & 0.1158358 & 0.1143157 \\
& $(0.046148)$ & $(0.005846)$ & $(0.0057101)$ & $(0.0041457)$ & $(0.0018498)$ & $(0.0046383)$ \\
& {$[17.37]$} & {$[18.16]$} & {$[18.14]$} & {$[18.43]$} & {$[62.62]$} & {$[24.65]$} \\
idade^2 & -0.0007867 & -0.0010892 & -0.0000767 & -0.0008778 & -0.0012448 & -0.0012939 \\
& $(0.049023)$ & $(0.0000608)$ & $(0.0000608)$ & $(0.0000564)$ & $(0.0012448)$ & $(0.0000625)$ \\
& {$[-16.72]$} & {$[-17.72]$} & {$[-14.38]$} & {$[-15.57]$} & {$[-40.71]$} & {$[-20.71]$} \\
branco & 0.2160024 & 0.2342141 & 0.136875 & 0.096363 & 0.1918853 & 0.2475348 \\
& $(0.000613)$ & $(0.0273499)$ & $(0.0226211)$ & $(0.0078044)$ & $(0.0164036)$ & $(0.00136974)$ \\
& {$[12.83]$} & {$[8.56]$} & {$[6.00]$} & {$[12.35]$} & {$[11.70]$} & {$[18.07]$} \\
fem & -0.3675455 & -0.465766 & -0.36110987 & -0.1159613 & -0.686757 & -0.9297767 \\
& $(0.0205633)$ & $(0.389911)$ & $(0.0166585)$ & $(0.0108324)$ & $(0.020116)$ & $(0.379097)$ \\
& {$[-17.87]$} & {$[-11.95]$} & {$[-21.68]$} & {$[-10.71]$} & {$[-34.14]$} & {$[-24.53]$} \\
cons & 3.67996 & 1.295334 & 2.894311 & 3.860747 & 2.484523 & 2.808869 \\
& $(0.0964963)$ & $(0.1099442)$ & $(0.1146532)$ & $(0.0851216)$ & $(0.0499158)$ & $(0.0379097)$ \\
& {$[38.72]$} & {$[11.78]$} & {$[25.21]$} & {$[45.36]$} & {$[77.99]$} & {$[32.31]$} \\
\hline
\end{tabular}

\begin{tabular}{|c|c|c|c|c|c|c|}
\hline \multicolumn{7}{|c|}{ Q25 } \\
\hline Lnw & DIR & PCA & TME & SAD & SERV & TPROD \\
\hline \multirow{4}{*}{ escolaridade } & 0.0916655 & 0.1711424 & 0.0884703 & 0.0552293 & 0.060347 & 0.0603047 \\
\hline & (0.01964) & (0.001324) & (0.0055483) & $(0.0510762)$ & $(0.0012727)$ & $(0.0012727)$ \\
\hline & [46.67] & [129.26] & [25.18] & [25.37] & [47.38] & [45.17] \\
\hline & 0.0741891 & 0.0977883 & 0.083081 & 0.0510762 & 0.0963816 & 0.0963816 \\
\hline \multirow[t]{3}{*}{ idade } & $(0.0052999)$ & $(0.0004912)$ & $(0.00326871)$ & $(0.0000445)$ & (0.0018157) & $(0.000216)$ \\
\hline & [14.00] & [19.67] & [25.18] & [14.86] & [53.08] & [29.58] \\
\hline & -0.0006799 & -0.00964 & -0.0008057 & -0.0004979 & -0.0010084 & -0.0010084 \\
\hline \multirow[t]{3}{*}{ idade^2 } & (0.000683) & $(0.0000603)$ & $(0.0000379)$ & $(0.0101522)$ & $(0.007925)$ & $(0.000216)$ \\
\hline & {$[-9,96]$} & {$[-16.00]$} & {$[-21.27]$} & [-11.20] & [-46.79] & {$[-24.27]$} \\
\hline & 0.262313 & 0.2527723 & 0.1934318 & 0.1142131 & 0.1222229 & 0.1222229 \\
\hline \multirow[t]{3}{*}{ branco } & $(0.0262331)$ & $(0.0186122)$ & (0.0152031) & (0.0101942) & (0.0085913) & (0.001925) \\
\hline & [10.59] & [13.58] & [12.72] & [11.25] & [15.42] & [39.69] \\
\hline & -0.03543719 & -0.5232807 & -0.4317777 & -0.1360694 & -0.473839 & -0.473839 \\
\hline \multirow[t]{3}{*}{ fem } & $(0.0173586)$ & (0.173096) & (0.086035) & $(0.0673593)$ & (0.008913) & $(0.0085913)$ \\
\hline & {$[-20.41]$} & {$[-30.23]$} & {$[-50.19]$} & {$[-13.35]$} & {$[-55.15]$} & [-40.48] \\
\hline & 3.961564 & 2.129898 & 3.521501 & 4.446263 & 3.370113 & 3.370113 \\
\hline \multirow[t]{2}{*}{ cons } & $(0.0901252)$ & $(0.0933594)$ & (0.0752518) & $(0.0673593)$ & $(0.0478146)$ & $(0.0478146)$ \\
\hline & {$[4.00]$} & [22.80] & [46.80] & [66.01] & [70.48] & [92.71] \\
\hline \multicolumn{7}{|c|}{ Q50 } \\
\hline \multirow[t]{2}{*}{ Lnw } & DIR & PCA & TME & SAD & SERV & TPROD \\
\hline & 0.0995138 & 0.1730083 & 0.1019373 & 0.073351 & 0.0414893 & 0.0544163 \\
\hline \multirow[t]{3}{*}{ escolaridade } & (0.024964) & (0.004441) & (0.0030428) & $(0.025415)$ & $(0.0015957)$ & $(0.0012047)$ \\
\hline & [39.86] & [38.96] & [33.50] & [25.37] & [26.00] & [45.17] \\
\hline & 0.069348 & 0.0855909 & 0.078936 & 0.0566571 & 0.0635384 & 0.0724137 \\
\hline \multirow[t]{3}{*}{ idade } & $(0.0084586)$ & (0.005093) & $(0.0038156)$ & $(0.002448)$ & $(0.0022462)$ & $(0.0024484)$ \\
\hline & {$[8.20]$} & {$[16.81]$} & [20.69] & [14.86] & [28.29] & [29.58] \\
\hline & -0.0005589 & -0.0007873 & -0.0007114 & -0.0004835 & -0.0006545 & -0.0007566 \\
\hline \multirow[t]{3}{*}{ idade 2} & (0.001053) & $(0.0000588)$ & $(0.0000507)$ & $(0.0000365)$ & $(0.00027)$ & $(0.0000312)$ \\
\hline & {$[-5.31]$} & {$[-13.38]$} & {$[-14.04]$} & [-11.20] & {$[-24.21]$} & [-24.27] \\
\hline & 0.2745268 & 0.24764119 & 0.2169714 & 0.13730609 & 0.1046139 & 0.1888183 \\
\hline \multirow{3}{*}{ branco } & $(0.0168304)$ & $(0.024586)$ & (0.0162166) & $(0.0099726)$ & (0.0036201) & $(0.0047575)$ \\
\hline & [16.31] & [10.07] & [13.38] & [11.25] & [28.90] & [39.69] \\
\hline & -0.3597322 & -0.5800859 & -0.48497 & -0.1960375 & -0.3290699 & -0.429965 \\
\hline \multirow{3}{*}{ fem } & (0.0138924) & (0.389911) & (0.0109673) & $(0.0070263)$ & (0.006301) & $(0.0105719)$ \\
\hline & {$[-25.89]$} & {$[-25.68]$} & {$[-44.22]$} & {$[-13.35]$} & {$[-52.23]$} & [-40.48] \\
\hline & 4.331408 & 2.841099 & 3.777058 & 4.343475 & 4.483083 & 4.366563 \\
\hline \multirow[t]{2}{*}{ cons } & (0.1815645) & $(0.0968139)$ & $(0.0722442)$ & $(0.0400204)$ & $(0.0487886)$ & $(0.0471014)$ \\
\hline & {$[23.86]$} & [11.78] & [56.28] & [108.53] & [91.89] & [92.71] \\
\hline
\end{tabular}


TABELA 4 (CONTINUAÇÃO) - RESULTADO DAS REGRESSÕES QUANTÍLICAS POR OCUPAÇÃO

\begin{tabular}{|c|c|c|c|c|c|c|}
\hline \multicolumn{7}{|c|}{ Q75 } \\
\hline Lnw & DIR & PCA & TME & SAD & SERV & TPROD \\
\hline \multirow{4}{*}{ escolaridade } & 0.0952919 & 0.1701955 & 0.1095602 & 0.0896419 & 0.049186 & 0.0589336 \\
\hline & (0.031906) & (0.0037467) & (0.0030753) & $(0.02025278)$ & $(0.0011841)$ & (0.0011539) \\
\hline & [29.87] & [45.42] & [35.63] & [35.46] & [41.54] & [51.07] \\
\hline & 0.069392 & 0.0896022 & 0.0863353 & 0.0611812 & 0.053 & 0.0723793 \\
\hline \multirow[t]{3}{*}{ idade } & (0.0053115) & $(0.0073129)$ & $(0.0047879)$ & $(0.0023372)$ & $(0.0016965)$ & (0.0018527) \\
\hline & [12.98] & [12.25] & [18.03] & [26.18] & [31.24] & [39.07] \\
\hline & -0.0005102 & -0.0007949 & -0.000741 & -0.00044 & -0.0005144 & -0.00007076 \\
\hline \multirow[t]{3}{*}{$\operatorname{idade}{ }^{\wedge} 2$} & $(0.000587)$ & $(0.00085)$ & $(0.0000726)$ & $(0.000034)$ & $(0.00027)$ & (0.0000253) \\
\hline & {$[-8.69]$} & {$[-9.35]$} & {$[-10.21]$} & {$[-12.93]$} & {$[-27.48]$} & {$[-28.02]$} \\
\hline & 0.2376618 & 0.2306564 & 0.2187523 & 0.16119 & 0.1306293 & 0.1916198 \\
\hline \multirow[t]{3}{*}{ branco } & $(0.0375317)$ & $(0.0242716)$ & (0.0119837) & (0.0095778) & (0.000187) & (0.0076409) \\
\hline & [6.33] & [9.50] & [18.25] & [16.83] & [29.56] & [25.08] \\
\hline & -0.3543719 & -0.5800859 & -0.4872204 & -0.2528272 & -0.3290699 & -0.4506693 \\
\hline \multirow[t]{2}{*}{ fem } & (0.0173586) & (0.0294966) & $(0.0139029)$ & (0.0085223) & $(0.0044188)$ & $(0.0085156)$ \\
\hline & $\begin{array}{r}{[-20.41]} \\
4830400\end{array}$ & [-19.94] & {$\left[\begin{array}{r}-35.04] \\
30107\end{array}\right.$} & {$[-29.67]$} & [-46.03] & [-52.92] \\
\hline \multirow{2}{*}{ cons } & $\begin{array}{r}4.839409 \\
(0.1166721)\end{array}$ & $\begin{array}{r}3.2 / 7899 \\
(0.1147231)\end{array}$ & $\begin{array}{r}3.9197 \\
(0.1008949)\end{array}$ & $\begin{array}{r}4.317614 \\
(0.043688)\end{array}$ & $\begin{array}{r}4.890833 \\
(0.0416134)\end{array}$ & $\begin{array}{r}4.598808 \\
(0.0352393)\end{array}$ \\
\hline & [41.48] & [28.57] & [38.85] & [98.36] & [117.53] & [130.50] \\
\hline \multicolumn{7}{|c|}{ Q90 } \\
\hline \multirow[t]{2}{*}{ Lnw } & DIR & PCA & TME & SAD & SERV & TPROD \\
\hline & 0.0879536 & 0.1601009 & 0.1080421 & 0.0968532 & 0.049186 & 0.0655671 \\
\hline \multirow[t]{3}{*}{ escolaridade } & $(0.047665)$ & (0.0040188) & (0.0041188) & (0.023133) & $(0.0011841)$ & (0.0018623) \\
\hline & [18.45] & [39.84] & [26.23] & [41.87] & [41.54] & [35.21] \\
\hline & 0.0611089 & 0.0946734 & 0.0885217 & 0.0765963 & 0.0544592 & 0.0799282 \\
\hline \multirow[t]{3}{*}{ idade } & (0.0070547) & $(0.0051969)$ & (0.0061485) & $(0.003236)$ & $(0.0016965)$ & (0.0020786) \\
\hline & [8.66] & [18.22] & [14.40] & [23.67] & [24.50] & [38.45] \\
\hline & -0.000389 & -0.0008169 & -0.0007139 & -0.000567 & -0.0004808 & -0.0000269 \\
\hline \multirow[t]{3}{*}{ idade^2 } & $(0.000389)$ & (0.0000608) & $(0.0000831)$ & $(0.00467)$ & $(0.0018088)$ & $(0.0099811)$ \\
\hline & {$[-4,64]$} & {$[-13.45]$} & {$[-8.59]$} & {$[-12.13]$} & {$[-16.37]$} & [-27.88] \\
\hline & 0.1957726 & 0.2216822 & 0.2331365 & 0.13433911 & 0.1805924 & 0.1967013 \\
\hline \multirow[t]{3}{*}{ branco } & (0.033634) & (0.0172966) & (0.0150668) & $(0.0148759)$ & (0.000294) & (0.0099811) \\
\hline & [5.82] & [12.82] & [15.47] & [9.03] & [20.05] & [19.71] \\
\hline & -0.3565806 & -0.5446986 & -0.4684177 & -0.2800733 & -0.406632 & -0.4837527 \\
\hline \multirow[t]{3}{*}{ fem } & (0.0422118) & (0.0191381) & (0.0150668) & (0.0167984) & $(0.0091992)$ & $(0.120054)$ \\
\hline & {$[-8.45]$} & [-28.46] & {$[-25.72]$} & {$[-16.67]$} & [-44.20] & [-40.29] \\
\hline & 5.570144 & 3.723506 & 4.23438 & 4.263448 & 4.960848 & 4.686807 \\
\hline \multirow[t]{2}{*}{ cons } & (0.1604954) & $(0.1125526)$ & $(0.1229649)$ & $(0.0546655)$ & $(0.0376957)$ & (0.0393617) \\
\hline & {$[34.71]$} & [33.08] & [34.44] & [77.99] & [131.60] & [119.07] \\
\hline
\end{tabular}

FONTE: Elaboração própria a partir de dados da PNAD de 2009.

NOTA: Entre parênteses apresentam-se os desvios-padrão e entre colchetes a estatística T.

Cabe destacar que a característica idade é a que menos contribui para o diferencial de rendimentos entre as diferentes ocupações. Apesar da pequena mudança na magnitude deste coeficiente ao longo dos quantis, pode-se afirmar que a idade torna-se menos importante nos quantis de renda mais elevados. Destaque-se ainda que a utilização da variável idade ao quadrado justifica-se pelo fato de que o efeito da idade sobre o rendimento tende a ser não linear.

Os resultados indicam que o gênero feminino contribui de modo negativo para determinar o rendimento em todas as ocupações, o que sugere a existência de preconceito de gênero no mercado de trabalho brasileiro. A ocupação dos Profissionais das Ciências e das Artes é aquela caracterizada por maior preconceito de gênero, apesar de que, no primeiro quantil de renda, os coeficientes ligados a esta variável são extremamente elevados nas ocupações dos 
Trabalhadores dos Serviços e Trabalhadores da Produção. Nesse caso, a explicação pode estar no fato de que muitas profissões incluídas nessas ocupações são, em geral, masculinas.

Outro tipo de preconceito no mercado de trabalho brasileiro é o preconceito de cor, isto porque o fato de ser branco mostra-se como um aspecto que sempre contribui positivamente para o rendimento em todas as ocupações e percentis de renda. As ocupações caracterizadas por maior rendimento médio, Dirigentes e Profissionais das Ciências e das Artes, são aquelas nas quais se observa o maior preconceito racial. O coeficiente relacionado à variável branco é menor na ocupação Serviços Administrativos nos primeiros e segundos quantis; na ocupação Trabalhadores dos Serviços, no terceiro e quarto e, na categoria Trabalhadores da Produção, no último quantil.

\section{CONSIDERAÇÕES FINAIS}

Este trabalho avaliou empiricamente a problemática dos diferenciais de salários no mercado de trabalho brasileiro sob a perspectiva da Teoria da Segmentação, buscando apreender a presença destes diferenciais a partir de dois procedimentos: segundo as características de cada grupo e, em seguida, por grupos ocupacionais selecionados.

Investigou-se, primeiramente, a presença dos diferenciais de salários, considerando os fatores históricos, consubstanciados na natureza de cada grupo de ocupação selecionado. Neste caso, entende-se que os diferenciais de salários resultam das condições históricas, que conformam cada um dos segmentos do mercado de trabalho, na acepção da própria Teoria da Segmentação.

Deste modo, poderia ser dito que os diferenciais de salários assim estimados refletem as descontinuidades do mercado de trabalho consubstanciadas na segmentação deste mercado em primário independente ou criativo, primário dependente ou rotineiro e secundário.

Se tal afirmação for plausível, a estimativa realizada sugere que os grupos ocupacionais DIR e PCA sejam próprios do segmento primário independente, enquanto as categorias TMED e SADM sejam próprias do primário dependente. Os outros dois grupos ocupacionais, SERV e TPROD, parecem mais próprios dos segmentos primário dependente e secundário.

O segundo procedimento investigou a presença de diferenciais de salários, para os grupos ocupacionais selecionados, considerando características tais como cor, gênero, idade e escolaridade, as quais não se encontram na base da conformação dos segmentos do mercado de trabalho acima referida, buscando responder até que ponto cada grupo ocupacional pode apresentar diferencial de salários, quando exposto à tais características.

Neste caso, os diferenciais de salários refletem mais de perto a discriminação dos 
trabalhadores quanto à cor, idade e gênero e, mais remotamente, com relação à educação, que é reflexo dos valores sociais e culturais que permeiam o imaginário dos empregadores e que influencia as oportunidades de trabalho e as disparidades salariais nos intrassegmentos do mercado de trabalho.

Em quaisquer procedimentos, a educação perde o caráter de centralidade na discussão dos diferenciais de salários. É o que se constata da investigação econométrica, cujos resultados sugerem que: i) a educação é menos importante para determinar o rendimento naqueles segmentos de trabalho mais precários, quais sejam as categorias dos Trabalhadores dos Serviços e da Produção; ii) os coeficientes das variáveis cor e gênero apontam a existência de preconceito no mercado de trabalho, que é maior nas categorias ocupacionais com maior potencial de rendimento, isto é, os Profissionais das Ciências e das Artes e os Dirigentes; e, por fim, iii) a variável idade é a menos significativa para determinar o rendimento nas diferentes ocupações.

Diante do exposto, cabe destacar que os resultados obtidos na presente análise sugerem a existência de uma relação não linear entre educação (ou investimento em capital humano) e rendimento, como sustentado pela Teoria do Capital Humano. Do contrário, pressupõe e implica a necessidade de que se considerem elementos ligados à natureza e ao processo histórico peculiar a cada país ou região como forma de apreender as causas e o sentido dessas relações.

\section{REFERÊNCIAS}

ALMEIDA, E. P.; PEREIRA, R. S. Críticas à teoria do capital humano: uma contribuição à análise de políticas públicas em educação. Texto para Discussão do IPEA, n. 863. Disponível em: <http://www.ipea.gov.br>.

BECKER, G. S. Human capital. New York: Columbia University Press, 1964.

CACCIAMALI, M.; BATISTA, N. Diferencial de salários entre homens e mulheres segundo a condição de migração. Revista Brasileira de Estudos Populacionais, São Paulo, v. 26, n. 1, p. 97-115, jan./jun. 2009.

HOFFMANN, R. Distribuição de renda: medidas de desigualdade e pobreza. São Paulo: Universidade de São Paulo, 1998. 275 p.

LIMA, R. Mercado de trabalho: o capital humano e a teoria da segmentação. Pesquisa e Planejamento Econômico, Rio de Janeiro, n. 10, p. 217-272, abr. 1980.

MACHADO, J. A. F.; MATA, J. Counterfactual decomposition of changes in wage distributions using quantile regression. Journal of Applied Econometrics, v. 20, n. 4, p. 445-465, 2005.

MINCER, J. Investment in human capital and personal income distribution. The Journal of Political Economy, v. 66, n. 4, p. 281-302, August 1958.

MULS, L. A teoria do capital humano, as teorias da segmentação e a literatura institucionalista: proposições de políticas públicas e implicações sobre distribuição de renda. In: ENCONTRO NACIONAL DE ECONOMIA POLÍTICA, 4., 1999, Porto Alegre. Anais... Porto Alegre: 
SEP, 1999. Disponível em: <http://www.sep.org.br/pt/artigo_old_list.php?id=4>.

SCHULTZ, T. W. Capital formation by education. Journal of Political Economy, v. 68, n. 6, p. 571-583, 1960.

SCHULTZ, T. W. Investments in human capital. American Economic Review, v. 51, n. 1, p. 1-17, 1961. 